\title{
Investigação geoelétrica de depósito de barita - resultados preliminares
}

\author{
Fabiana Pereira Lasmar, IAG/USP, Vagner Roberto Elis, IAG/USP
}

Copyright 2019, SBGf - Sociedade Brasileira de Geofísica

This paper was prepared for presentation during the $16^{\text {th }}$ International Congress of the Brazilian Geophysical Society held in Rio de Janeiro, Brazil, 19-22 August 2019.

Contents of this paper were reviewed by the Technical Committee of the $16^{\text {th }}$ International Congress of the Brazilian Geophysical Society and do not necessarily represent any position of the SBGf, its officers or members. Electronic reproduction or storage of any part of this paper for commercial purposes without the written consent of the Brazilian Geophysical Society is prohibited.

\section{Abstract}

A barita $\left(\mathrm{BaSO}_{4}\right)$ é um mineral industrial, utilizado principalmente na indústria petrolífera, no fluido de perfuração, e apresenta uma densidade elevada $\left(4,48 \mathrm{~g} / \mathrm{cm}^{3}\right)$ quando compardo aos silicatos. Na literatura brasileira há pouco registro de estudo geofísico na prospecção desse bem mineral. A princípio, devido a alta densidade desse sulfato, se propõe a utilização da gravimetria, mas em consequência da pequena espessura dos veios a resolução do método não é eficiente. Um estudo mostra que ensaios de resistividade podem localizar os corpos de barita e diferenciá-los da encaixante. Assim, esse trabalho propõe a utilização dos métodos elétricos de resistividade e polarização induzida em corpos mineralizados na região do Vale do Ribeira, para avaliar o potencial da geofísica na identificação do minério.

\section{Introdução}

A pesquisa geofísica de determinados bens minerais, como por exemplo a barita, tem sido pouco registrada na literatura. Esse mineral é um sulfato de Bário $\left(\mathrm{BaSO}_{4}\right)$, que pode ser encontrado em rochas ígneas, metamórficas ou sedimentares. É utilizado, principalmente, nos fluidos de perfuração de poços de petróleo, porém é empregado também, com menor relevância, na indústria química, de autopeças, metalurgia e siderurgia.

O presente estudo visa à aplicação de métodos geofísicos na prospecção de barita, que são bastante escassos na literatura especializada. Em razão da alta densidade desse mineral $\left(4,48 \mathrm{~g} / \mathrm{cm}^{3}\right)$ em relação aos silicatos (quartzo 2,65 $\mathrm{g} / \mathrm{cm}^{3}$, argila $2,60 \mathrm{~g} / \mathrm{cm}^{3}$ ), a princípio poderia se pensar em utilização de gravimetria, mas devido a pequena espessura dos veios a resolução do método não seria eficiente. Isso é relatado por Bhattachary et al. (1974), que também demonstrou que ensaios de resistividade possibilitam a detecção de veios de barita. Outros trabalhos mais recentes apresentam resultados promissores, porém apenas sugerem zonas potencialmente mineralizadas, sem a correlação com dados diretos (Oladapo, \& Adeoye-Oladapo, 2011; Ehirim et al. 2016).

A área de estudo se encontra na região do Vale do Ribeira, em Tunas (PR), a sudoeste do centro urbano, onde existem ocorrências de barita que foram lavradas artesanalmente e que com o auxílio de conhecimento geológico são passíveis de constituir uma pequena mineração.

A justificativa desse projeto é que a utilização de métodos geofísicos pode contribuir muito com a exploração de barita, não só na região de estudo, como também para outras regiões, visto que não se tem muitos estudos que determinam o método ideal para prospecção da barita.

O Vale do Ribeira está inserido no contexto geotectônico do Cinturão Ribeira, que de acordo com Hasui 2012, remonta sua evolução desde o Neoarqueano. A área de estudo se localiza no Grupo Setuva, de idade Proterozóico médio, na Formação Perau, que é caracterizada por Soares (1988, apud SILVA, 1990, p. 34) sendo um ambiente marinho profundo, pelo fato de apresentar sequências turbidíticas, cálcio-ferromanganesíferas e vulcânicas básicas submarinas.

A Mineropar (Serviço Geológico do Paraná) já publicou alguns relatórios que descrevem mineralizações de barita relacionadas à formação Perau, em Rio Branco do Sul e Bocaiúva do Sul. A figura 1 mostra o mapa geológico da região, baseado na folha de Curitiba, que descreve 0 membro hospedeiro da barita a ser estudada como: "Quartzitos puros, quartzitos micáceos e quartzo xistos, com frequentes impregnações de óxido de ferro, de granulometria média a fina, mal selecionados."

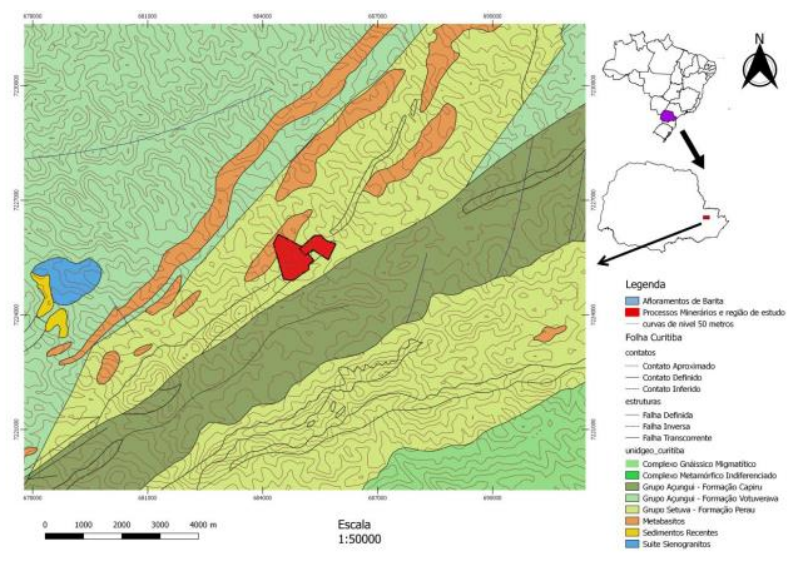

Figura1- Mapa geológico regional, baseado na folha de Curitiba. Destaque para área de estudo.

\section{Metodologia}

Como Bhattacharya et al. (1974) demonstraram que métodos gravimétricos não são eficientes para corpos de barita de pequena extensão, nesse estudo optou-se pelo uso de métodos elétricos. Esses métodos são passíveis 
de serem aplicados, devido à ocorrência de barita em rochas menos resistivas.

Os ensaios preliminares, realizados na área de estudo, tem o objetivo de avaliar a resposta geofísica em corpos mineralizados conhecidos. Os métodos utilizados, inicialmente, foram eletrorresistividade e polarização induzida, por meio da técnica de caminhamento elétrico dipolo-dipolo.

O caminhamento elétrico $(\mathrm{CE})$ é realizado através de perfis, estudando as variações laterais do parâmetro físico medido. A Figura 2 ilustra a disposição no campo, dos eletrodos de emissão de corrente e recepção de potencial, no desenvolvimento da técnica do CE - arranjo de campo dipolo-dipolo. Após a disposição do arranjo no terreno, fixados os níveis de investigação, e obtenção das leituras pertinentes, todo o arranjo é deslocado para a estaca seguinte e efetuadas as leituras correspondentes, continuando esse procedimento sucessivamente até atingir-se o final do perfil levantado.

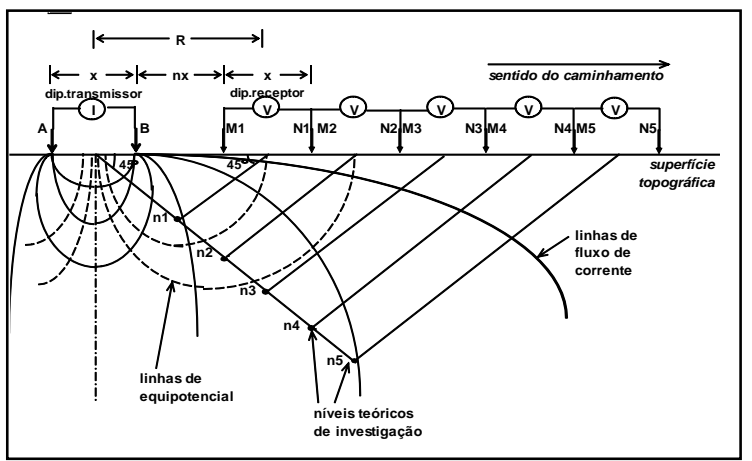

Figura 2- Disposição no campo do arranjo Dipolo Dipolo - técnica do Caminhamento Elétrico

A eletrorresistividade é um dos métodos mais utilizados em pesquisas de prospecção geofísica. Nele as investigações elétricas são realizadas aplicando uma corrente elétrica artificial no subsolo através de um ou dois eletrodos, denominados eletrodos de corrente A e B. Estes eletrodos são utilizados para medir o potencial gerado por outros eletrodos, chamados eletrodos de potencial $\mathrm{M}$ e $\mathrm{N}$. Com esta disposição de eletrodos é possível calcular a resistividade aparente das camadas no interior da Terra, a partir da equação:

$\rho_{a}=K . \frac{\Delta V}{I}$ (ohm.m)

$\mathrm{Na}$ equação $\rho$ representa a resistividade elétrica em ohm.m, K é o fator geométrico que depende da disposição do arranjo, $\Delta V$ é a diferença de potencial entre os eletrodos $\mathrm{M}$ e $\mathrm{N}$ e I é a intensidade de corrente que passa entre os eletrodos $\mathrm{A}$ e $\mathrm{B}$. $\mathrm{O}$ fator geométrico $\mathrm{K}$ pode ser obtido através da seguinte equação:

$$
K=2 \pi \cdot\left(\frac{1}{\overline{\mathrm{AM}}}-\frac{1}{\overline{\mathrm{BM}}}-\frac{1}{\overline{A N}}+\frac{1}{\overline{B N}}\right)^{-1}
$$

A Polarização Induzida (IP) é um fenômeno elétrico estimulado por corrente elétrica observado como resposta retardada à voltagem, em materiais naturais (Sumner, 1976). Em alguns corpos que são atravessados por uma corrente elétrica, quando esta corrente é cortada, o campo elétrico não desaparece imediatamente e sim de um modo mais lento. Esta é a chamada polarização induzida ou residual, de magnitude bastante reduzida, sendo na prática medida tal como uma variação de voltagem em função do tempo (IP domínio do tempo) ou frequência (IP domínio da frequência). No IP domínio do tempo uma diferença de pontencial $\Delta V$ primária ao solo é provocada e, consequentemente, uma polarização deste. $\mathrm{A}$ diferença de pontencial primária $\left(\Delta \mathrm{V}_{\mathrm{IP}}\right)$ varia com o tempo na forma de uma curva $\Delta \mathrm{V}_{\mathrm{IP}}=\mathrm{f}(\mathrm{t})$, como mostra a figura 3 . O fenômeno se observa quando se faz passar corrente elétrica através de um eletrólito que contém corpos ou partículas que possuem condutividade eletrônica (metais, semicondutores). A aquisição de dados de IP é muito semelhante às de resistividade aparente.

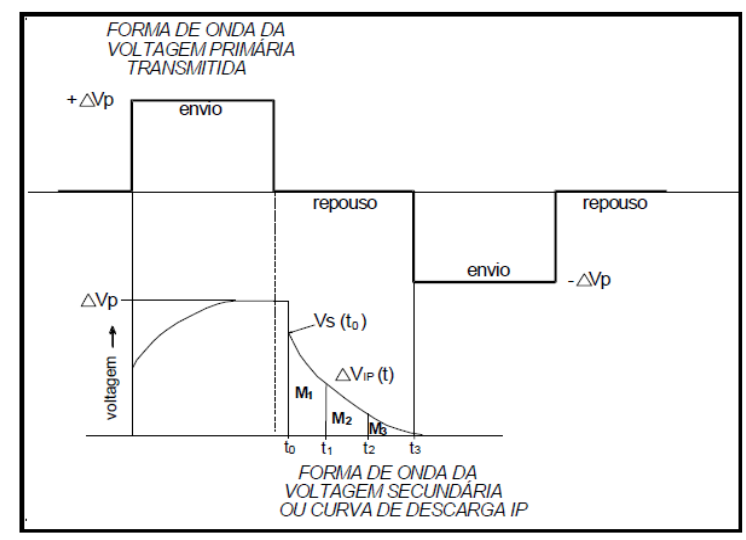

Figura 3- Variação de $\Delta V$ com o tempo. A linha pontilhada vertical indica o momento em que se corta a corrente e começa a descarga (Braga, 1997).

Silva (1990) descreve os veios de barita inseridos em quartzitos e filitos, sendo alguns deles aflorantes ou em pouca profundidade. Um mapa dos afloramentos de barita é mostrado na figura 4 .

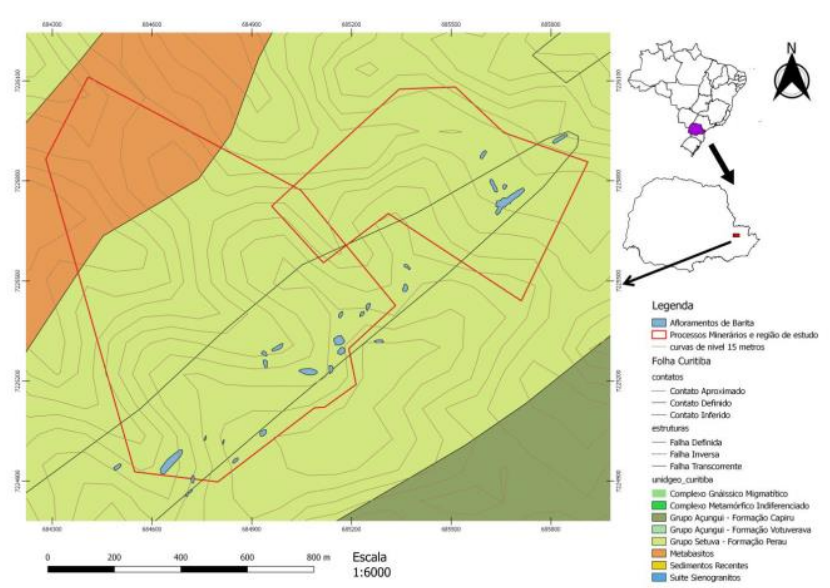

Figura 4- Mapa geológico da região de estudo baseado na folha de Curitiba. Destaque para afloramentos de barita. 
Os ensaios geofísicos preliminares foram realizados em dois locais, um com ocorrência conhecida (linha 1) e outra com ocorrência suspeita (linha 2), como mostrado no mapa da figura 5. No corpo conhecido, os ensaios de CE foram realizados primeiramente com arranjo dipolodipolo e abertura de 5 metros entre os eletrodos, tanto para eletrorresistividade quanto para IP. Para obtenção de melhor detalhe dessa região, foi feito um levantamento, no mesmo prefil, com aberturas menores, de 2 metros.

No local com suspeita de ocorrência de barita foi realizado um ensaio com abertura de 10 metros, com o objetivo de localizar zonas muito resistivas que possam indicar a presença de barita, também foram tomadas medidas de eletrorresistividade e IP.

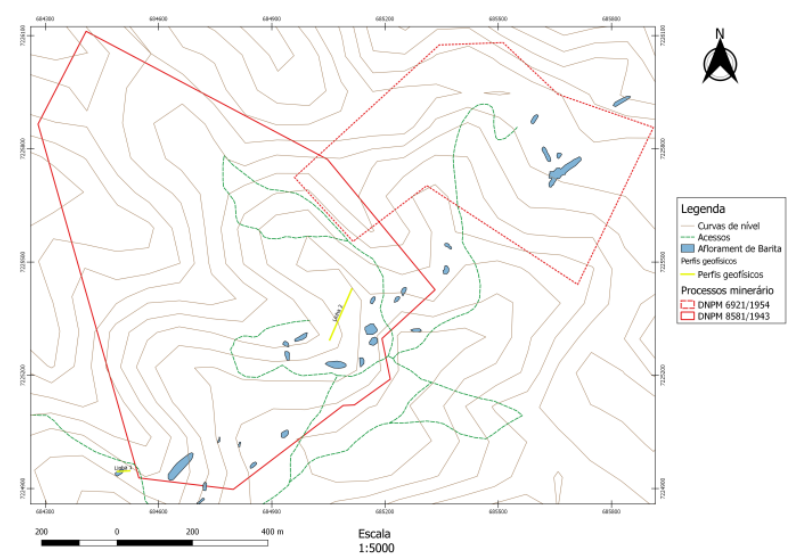

Figura 5- Mapa com afloramento de barita e perfis realizados.

\section{Resultados}

O resultado dos ensaios realizados sobre a linha $1 \mathrm{com}$ abertura de 5 metros é apresentado na figura 6. Observase uma zona de altíssima resistividade e baixa cargabilidade nas posições entre 13 e 20 metros (contorno tracejado preto), o que corrobora com as informações de campo, onde se observou o corpo de barita na posição de 14 metros e com espessura de cerca de 1 metro.

$\mathrm{Na}$ mesma linha 1, porém com abertura de 2 metros, observa-se com melhor definição o corpo mais resistivo da posição de 14 metros e com possíveis ramificações (até cerca de 4 metros) e lateralmente até a posição de 20 metros (figura 7). A linha tracejada preta representa toda a zona de maior resistividade, e da mesma forma que o perfil anterior, esses corpos resistivos apresentam baixa cargabilidade.

No local com suspeita de ocorrência de barita, o perfil obtido, apresentado na figura 8 , mostra duas zonas mais resistivas: uma na zona central do perfil, entre 80 e 90 metros, e outra muito resistiva no final do perfil. Essa zona no final do perfil apresenta valores de resistividade semelhantes aos da zona com barita na linha 1 . Em termos de cargabilidade, a anomalia tem o mesmo comportamento que o perfil da linha 1 - baixa cargabilidade, ao passo que no final do perfil a cargabilidade é mais alta.

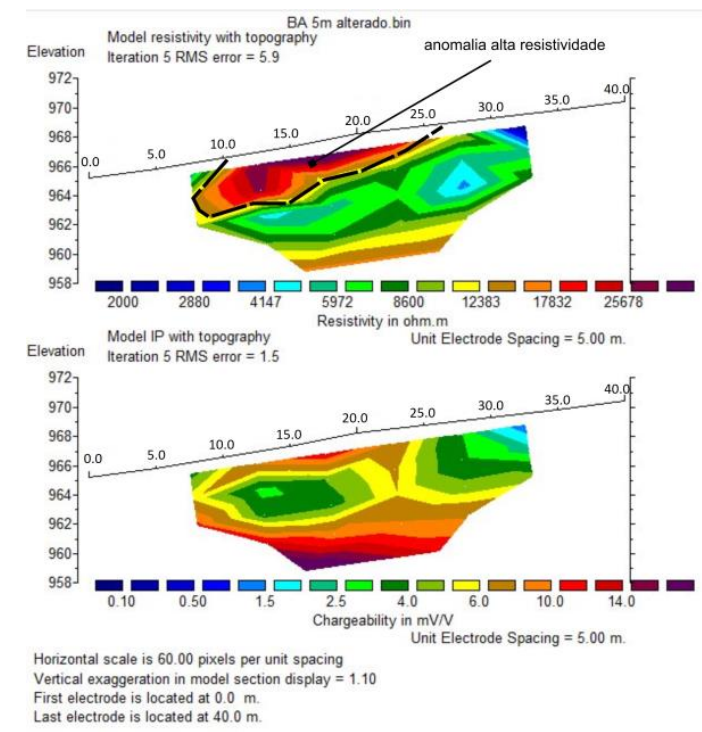

Figura 6 - Resultados eletrorresistividade e IP da Linha 1, abertura do dipolo 5 metros.

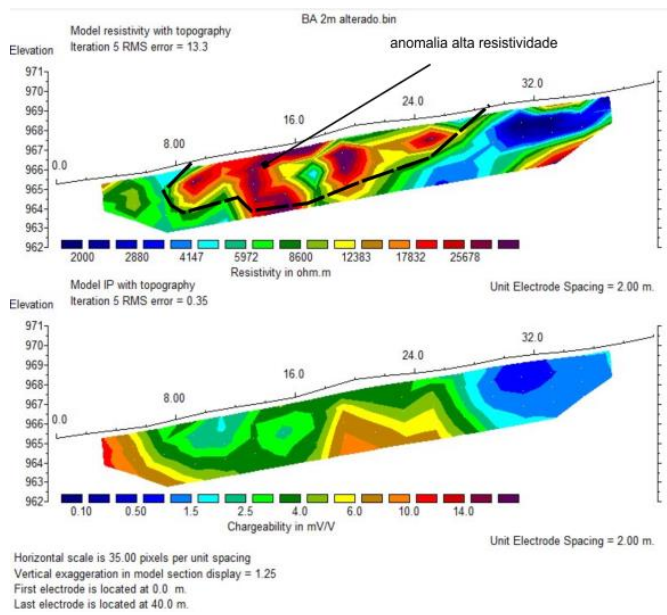

Figura 7- Resultados eletrorresistividade e IP da Linha 1, abertura do dipolo 2 metros.

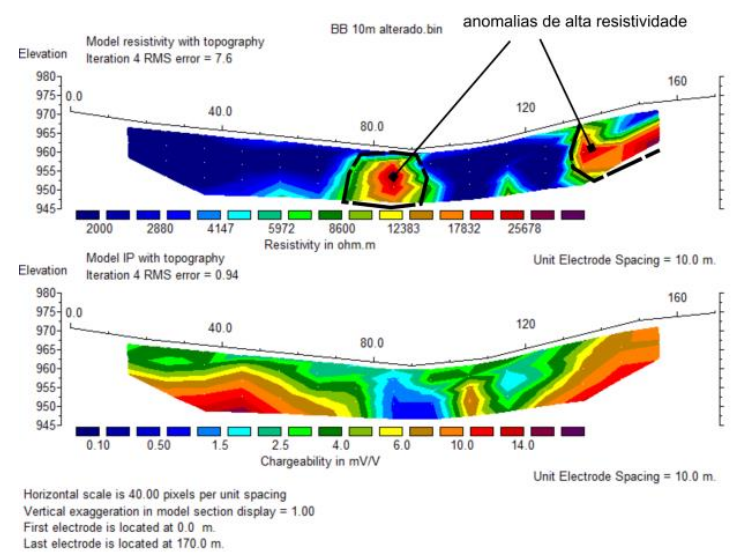

Figura 8- Resultados eletrorresistividade e IP da Linha 2, abertura do dipolo 10 metros. 


\section{Conclusões}

$\mathrm{Na}$ figura 6, a zona de alta resistividade, delimitada pela linha tracejada preta, indica a zona resistiva de ocorrência da barita, que corrobora com as observações em campo. A zona de barita apresenta baixos valores de cargabilidade.

Nos ensaios com abertura do dipolo de 2 metros (figura 7), a linha tracejada preta também delimita a zona da barita (mais resistivo). Nesse resultado, é possível observer possíveis ramificações em profundidade, e é importante ressaltar que o contorno tracejado abrange vários corpos resistivos, que indica, possivelmente, corpos menores, o que é compatível com a observação em campo.

As duas zonas mais resistivas na linha 2 (figura 8), são zonas de possível ocorrência da barita, sendo que a zona no final do perfil, por apresentar valores de resistividade semelhantes aos da zona com barita na linha 1, é o local mais indicado para o prosseguimento da prospecção com métodos diretos. A resistividade relativamente mais baixa da encaixante é compatível com filitos que devem ocorrer nesse perfil.

Os resultados obtidos nos testes preliminares mostram que a metodologia, com a configuração de eletrodos utilizada, pode obter sucesso na identificação de corpos de barita.

\section{Agradecimentos}

O presente trabalho foi realizado com apoio da Coordenação de Aperfeiçoamento de Pessoal de Nível Superior - Brasil (CAPES) - Código de Financiamento 001 e do Instituo de Astronomia, Geofísica de Ciências Atmosféricas da Universidade de São Paulo.

\section{References}

BATOLLA JR., F. et al. Projeto Leste do Paraná - Folha de Curitiba. São Paulo : CPRM, 1977. Escala 1:100.000.

BHATTACHARYA B. B., JAIN, S.C and MELLICK, K. 1974 - Geophysical Prospecting for Barite. Geophysical Prospecting 22, pp. 421-429.

BRAGA, A.C.O. Métodos Geoelétricos aplicados na caracterização geológica e geotécnica - Formação Rio Claro e Corumbataí, no município de Rio Claro -SP. Tese (Geociências) - Instituto de Geociências e Ciências Exatas, UNESP, Rio Claro.

EHIRIM, C. N.; EBENIRO J. O.; OFOEGBU, C. O. - 2016 - Baryte mineral exploration in parts of the lower Benue Trough, Nigeria. International Journal of the Physical Sciences 11(21), pp. 279-286.

HASUI, Y., CARNEIRO, C.D.R., ALMEIDA, F.F.M. e BARTORELLI, A. - 2012 - Geologia do Brasil. São Paulo: Beca-BALL.

OLADAPO, M.I., ADEOYE-OLADAPO, O.O. - 2011 Geophysical investigation of barite deposit in Tunga,
Northeastern Nigeria. International Journal of the Physical Sciences 6(20), pp. 4760-4774.

SILVA, A.C.G.A. - 1990 - A jazida de barita de Água Clara no âmbito do precambriano do Vale do Ribeira, Estado do Paraná. Tese de Doutorado, Instituto de Geociências, Universidade de São Paulo. São Paulo-SP, 204 p.

SUMNER, J.S. - 1976 - Principles of Induced Polarization for Geophysical Exploration. Elsevier Scientific Publishing Company, Amsterdam, 277p. 